\title{
Color Reconstruction for CFA Images
}

\author{
Gwanggil Jeon \\ Department of Embedded Systems Engineering, Incheon National University \\ 119 Academy-ro, Yeonsu-gu, Incheon 406-772, Korea \\ gjeon@inu.ac.kr
}

\begin{abstract}
The color image enhancement is an important topic for last few decades and still many researchers are working and presenting their research findings in literature. There are two issues in digital camera, firstly color filtering and secondly low spatial resolution. To solve both issues, this paper presents a new super resolution approach. Although Bayer pattern CFA is widely used in camera industry, there are few alternatives such as X-Trans CFA pattern. As color restoration method for X-Trans CFA pattern is not many, this paper introduce filter based demosaicking method. We firsuly design filters then apply it to X-Trans CFA pattern. Then, full color images are obtained. Expeximental results indicate that the presented method provides satisfactory kesults.
\end{abstract}

Keywords: X-Trans, color filter array, super resolution, image processing

\section{Introduction}

The digital color images are generallo indicated by three color levels (red, green, and blue) at each pixel, and the camerasestimates three spectral measurements per each pixel [1-2]. This camera differentiates the light and displays it onto three single electronic sensors such as CCD or CMOS [3-6]. However, this array requests its appropriate driving electronics, and the corresponding three color images have to be registered accurately [78]. This process is time consuming work and causes additional costs. Therefore, most digital cameras adopt a single matrix assessing a single color per pixel [9]. The digital cameras have been attracting great favor with consumers and replaced their film-based cameras in various applications over the past few decades [10-14]. As a pixel contains only single colo information, the other two color information should be restored from the adjacent pixels, and this process is called demosaicking [15].

There are various copfigurations of sensor array, and Bayer color filter array (CFA) is one of the most well-known CFA patterns [16]. In this pattern, a sing pair has four pixels. Two pixels are obtained from green information and the other two are obtained from red and blue information. It is noted that the green information is arrayed in quincunx pattern.

There are several demosaicking methods that were presented to reduce the color artifaets with less computational time. Some of them are based on nearest neighbor, bilinear, or bicubic interpolation methods, which are assumed as simple methods. These methods are single channel based approaches. Although these methods are simple, their results images may contain color artifacts.

In this paper, we investigate X-Trans CFA pattern and its demosaicking algorithm. This paper is organized as follows. In Section 2, the proposed method is presented where $\mathrm{X}$-Trans CFA and its corresponding demosaicking method are explained. In Section 3, we explain the visual and objective performance comparison. Finally, Section 4 shows the concluding remarks. 


\section{Proposed Method}

The X-Trans CFA pattern has higher degree of randomness with an array of six-by-six pixel units which has 8 red pixels, 20 green pixels, and 8 blue pixels. This CFA pattern was presented by Fujifilm Company to try to lessen moiré and false color artifacts, and reconstruct high resolution images. Generally speaking, the Bayer pattern CFA uses antialiasing filters to reduce moiré effect. However, the X-Trans CFA sensor adopts inconsistent pattern, which make it more possible to reduce severe moiré and false color artifacts effect.

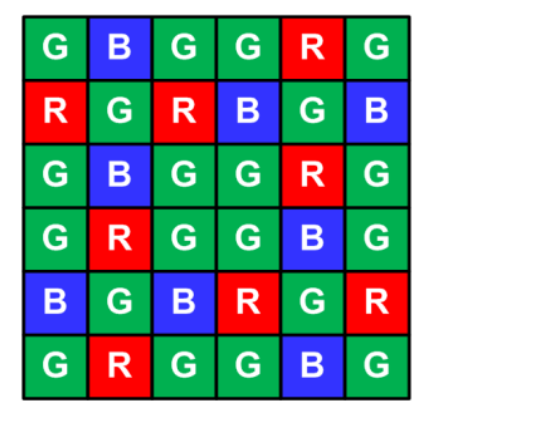

Figure 1. X-Trans CFA Pattern

Although the X-Trans CFA pattern is inconsistent (as can be, seen in Figure 1), all horizontal or vertical lines have at least one $\mathrm{R}, \mathrm{G}$, and $\mathrm{B}$ Cpizel. This is big difference between the Bayer CFA pattern and the X Trans CFA pattern, where horizontal or vertical lines do not have all three coloreomponents (B2yer CFA pattern). Another reason $\mathrm{X}$-Trans CFA pattern is superior to Baye CFA pattern is that, the X-Trans CFA pattern obtains more light than Bayer CFA pattern.

Figure 2 shows the process of color restoration for X-Trans CFA pattern. Figure 2 shows color restoration procedure for X-Trans CFA pattern. Figure 2(a) shown in the first column displays the X-Trans CFA pattern. Figure 2(b) shown in the second column displays three different color channels, red, green, and blue, where each six-by-six pair has 2:5:2 color components. This figure explains why green channel has more green information than that of Bayer patter CFA case (1:2:1). Figure 2(c) shown in the third column displays be reconstructed image. Finally, Figure 2(d) shown in the last column displays the full color reconstructed image.

Figure 3 shows actual $X$-Trans pattern in $128^{\text {th }}$ image, where 103 to 114 of $x$-axis, and 103 to 114 of $y$-axis are displayed. Figures 3(a) to 3(d) show red, green, blue, and CFA images. 

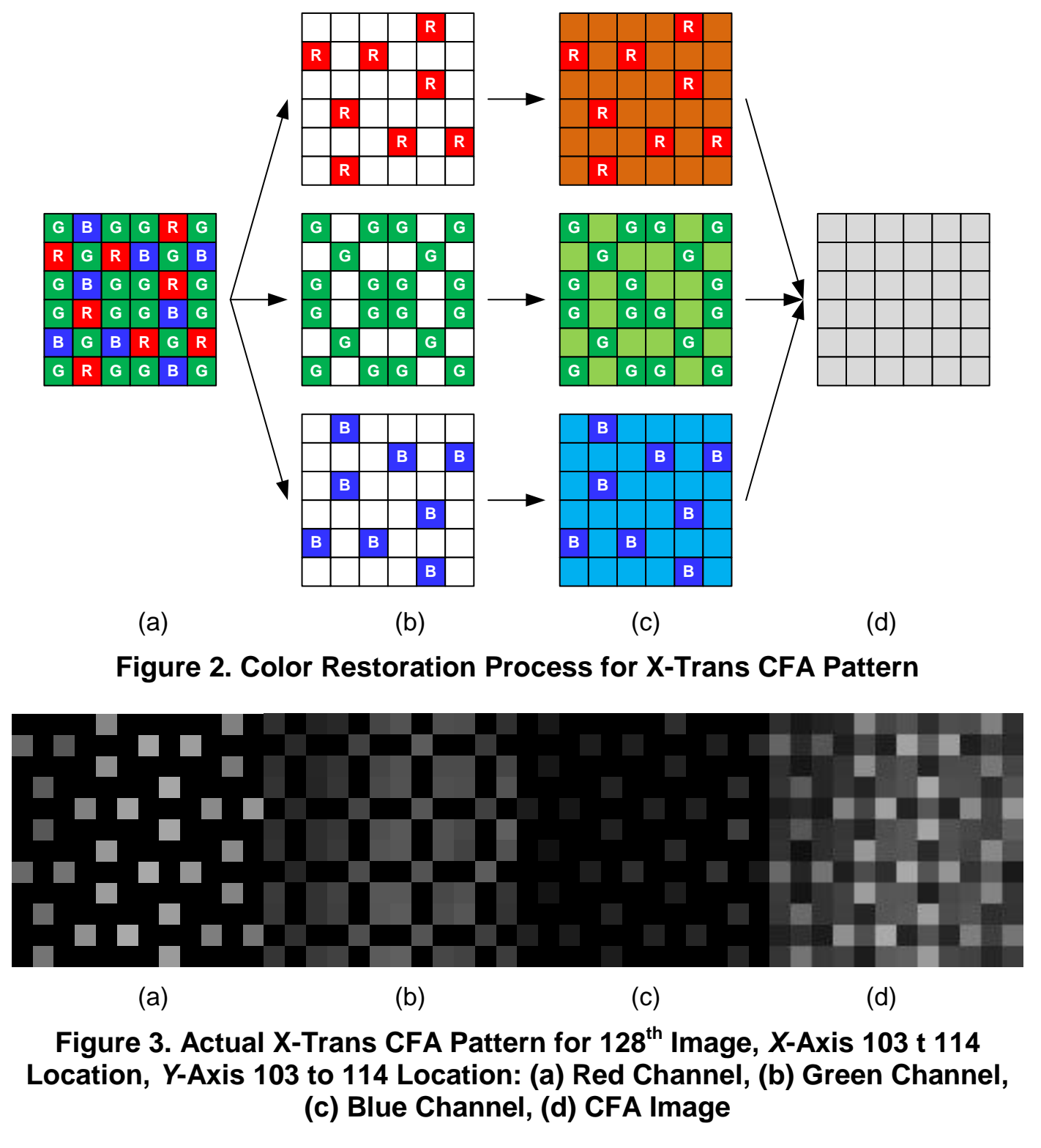

The proposed filter is designed by least squares method. The characteristics of linear time-invariant (LT) systems are mainly defined by their impulse response. The output of the LTI system to any input $x(i, j)$ can be determined via a convolution with the impulse response $h(i j j)$ :

$$
y(i, j)=x(i, j) * h(i, j)
$$

Above equation is spatial domain representation, which can be represented in frequency domain as shown in Eq. (2),

$$
Y(u, v)=X(u, v) H(u, v)
$$

Both equations indicate that the convolution of spatial domain is identical process of multiplication of frequency domain. This process can be used in several applications such as low-pass filter, band-pass filter, high-pass filter, and unsharp masking. To design these filters, we use standard least squares method. Our desired filter must be with minimized filter length, minimized error between original and the restored images, and minimized computational complexity.

The purpose of designing our filter is to obtain a filter $h$ that minimizes the errors, in other words, the output obtained by $h$ is the most approximately similar to the original one. 


$$
K h=H
$$

where $K$ is the Fourier matrix and $H$ is the frequency response. To solve this equation in a least squares sense, we calculate error cost as follows,

$$
\text { error }=|K h-H| \times|K h-H| \text {. }
$$

To solve this equation, one must minimize error cost with differentiate with respect to filter $h$,

$$
\frac{d}{d h} \text { error }=2 K^{T}|K h-H|
$$

This equation can be rearranged as

$$
2 K^{T}|K h-H|=2 K^{T} K h-2 K H
$$

Then, the filter $h$ is obtained as,

$$
h=\left(K^{T} K\right)^{-1} K^{T} H
$$

Filter $h$ is the result of the standard least squares form.

\section{Simulation Results}

In this section, we used 20 natural images of LC dataset, which has the size of $720 \times 540$ or $540 \times 720$. Out of 150 LC images, we selected $\$ 111-130$ images. The color PSNR is defined as follows:

where $\sigma$ is

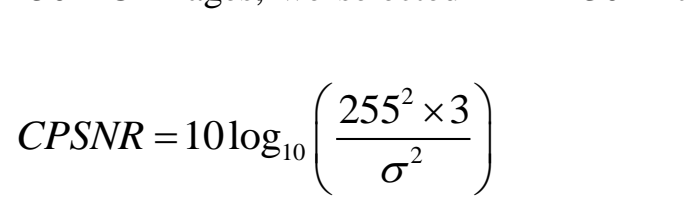

$$
\sigma^{2}=\frac{1}{N M} \sum_{i=0}^{N-1} \sum_{j=0}^{M-1}\left\{|R(i, j)-R(i)|-|G(i, j)-G(i, j)|^{2}+|B(i, j)-B(i, j)|^{2}\right\}
$$

and $M N$ is the size of an image A pixel of a color image can be assumed as a three dimensional vector

Figures 4-6 show 1D magnitude \& phase and 2D frequency response X-Trans CFA pattern of 128-130 LC images. Each figures in (a), (b) and (c) are red, green, and blue channel results. 

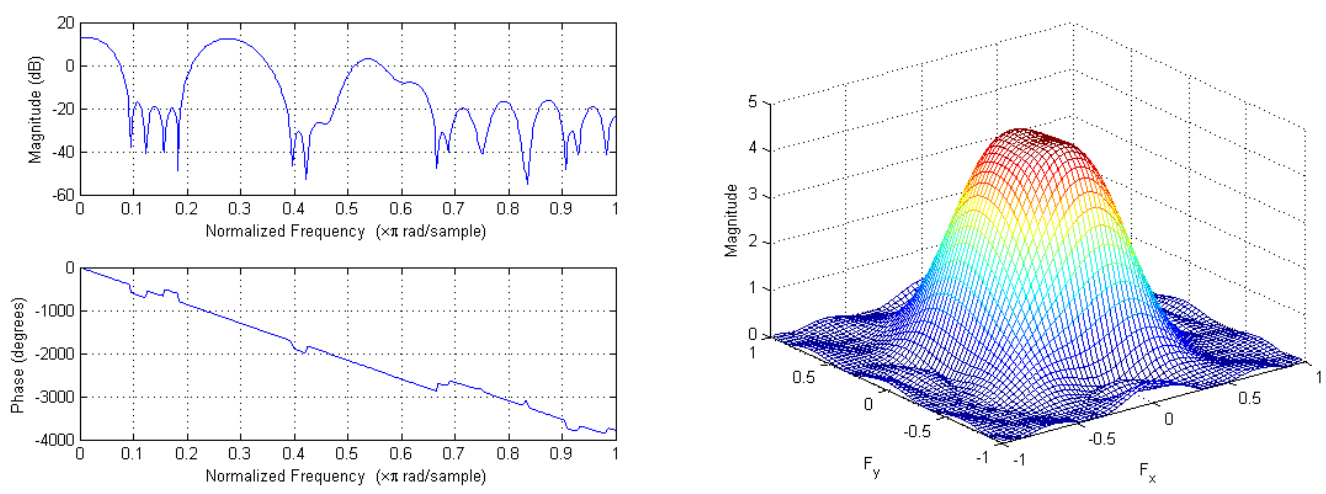

(a)
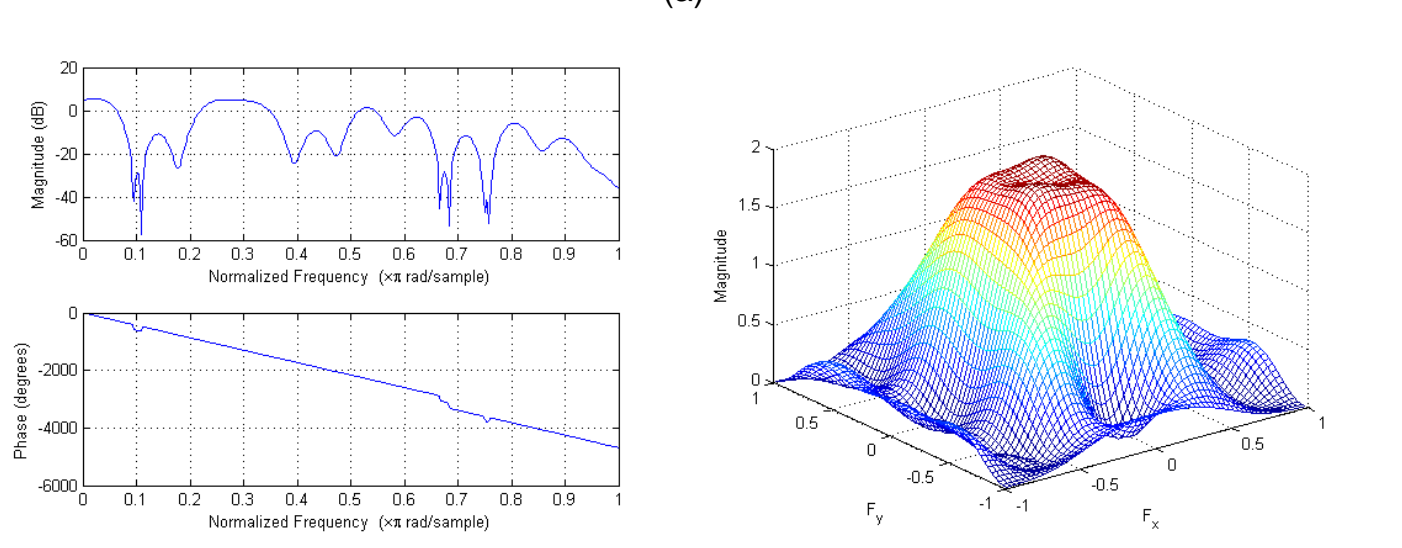

(b)

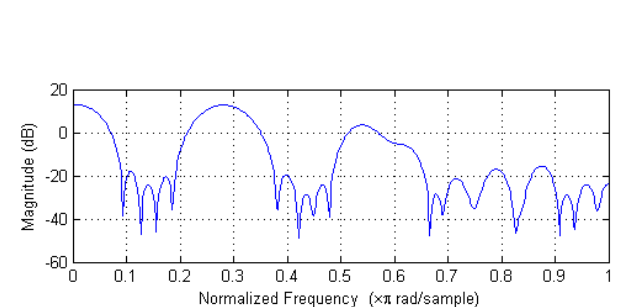

3
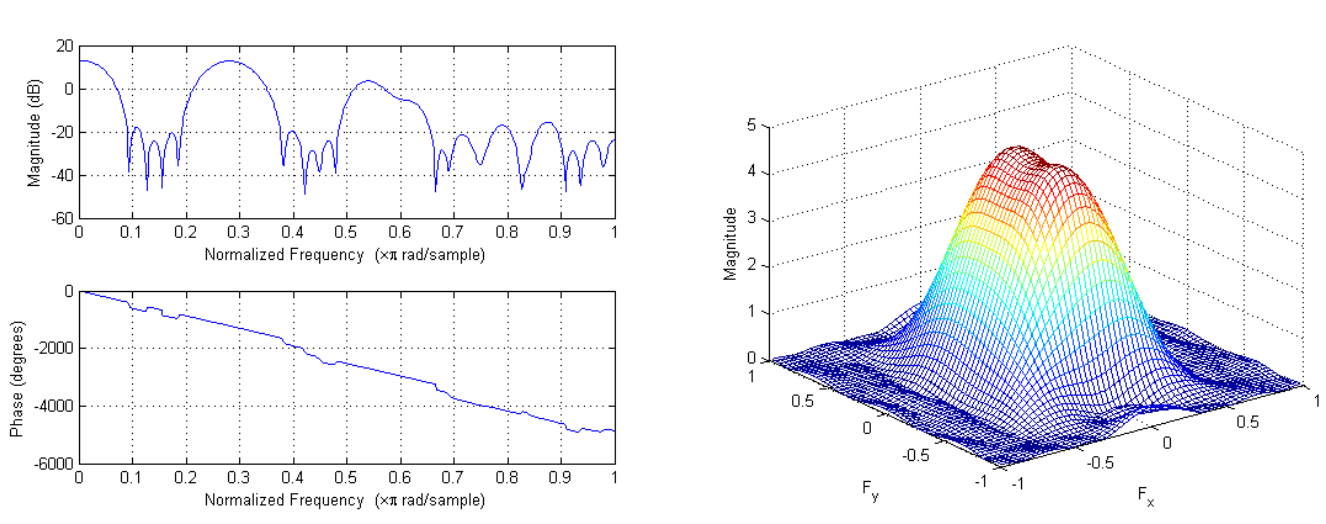

(c)

Figure 4. X-Trans CFA Pattern of $128^{\text {th }}$ LC Image, 1D Magnitude \& Phase and 2D Frequency Response: (a) Red Channel Filter, (b) Green Channel Filter, and (c) Blue Channel Filter 

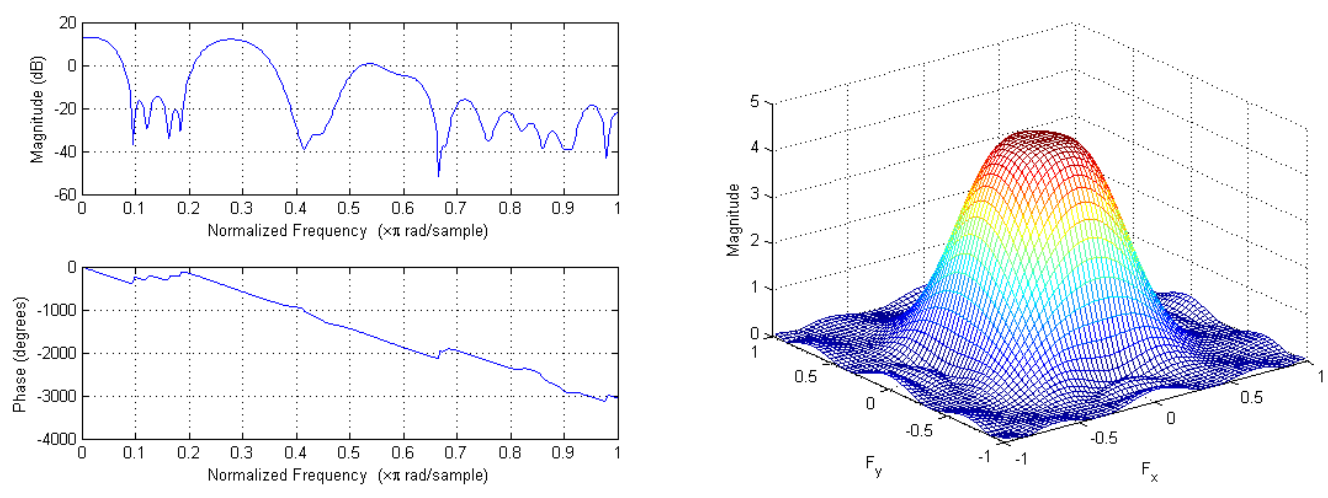

(a)
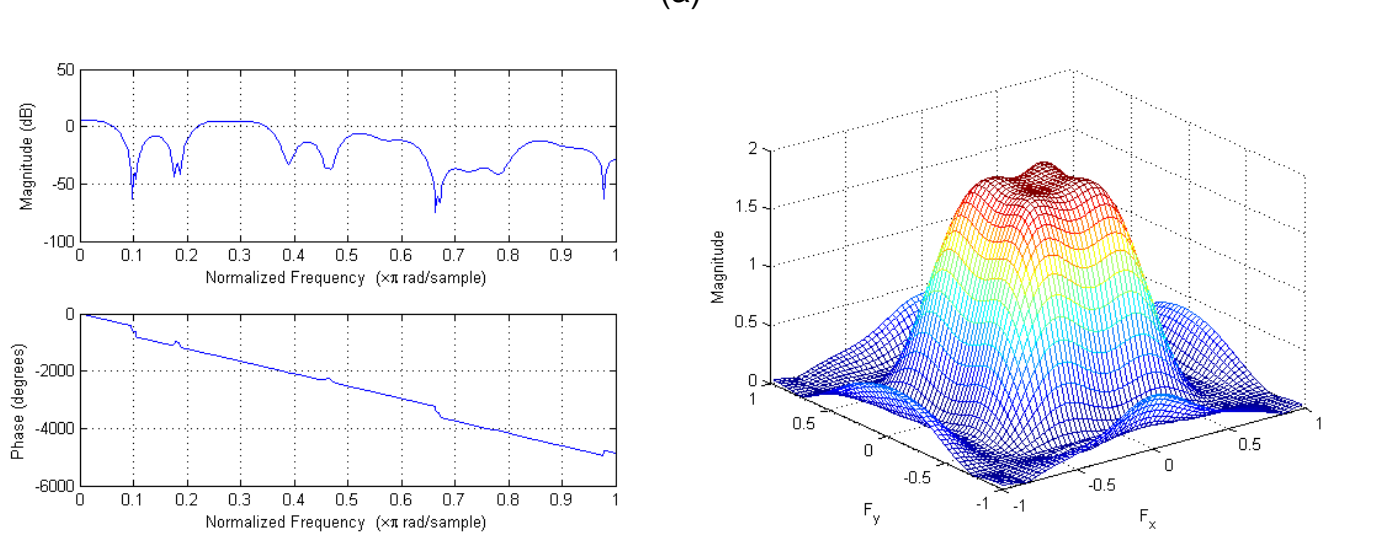

(b)

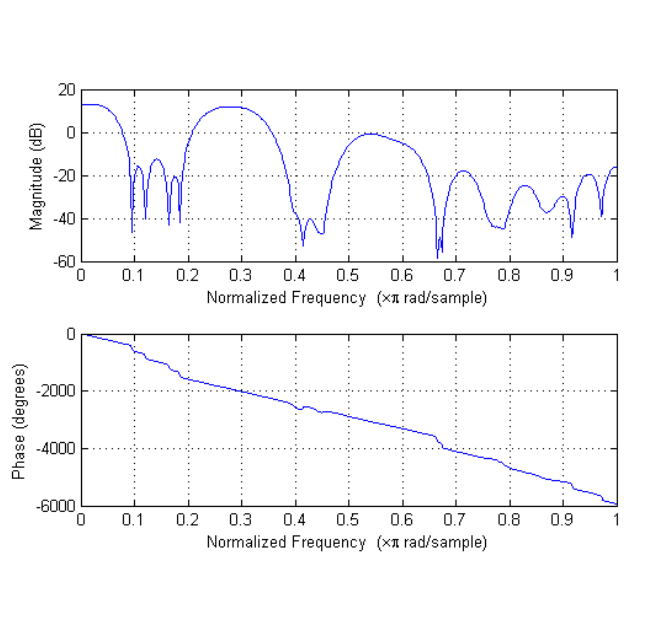

$\checkmark 2$

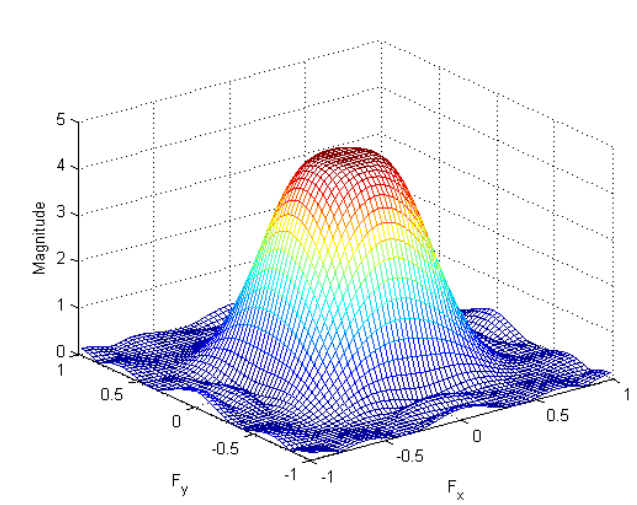

(c)

Figure 5. X-Trans CFA Pattern of $129^{\text {th }}$ LC Image, 1D Magnitude \& Phase and 2D Erequency Response: (a) Red Channel Filter, (b) Green Channel Filter, and (c) Blue Channel Filter 

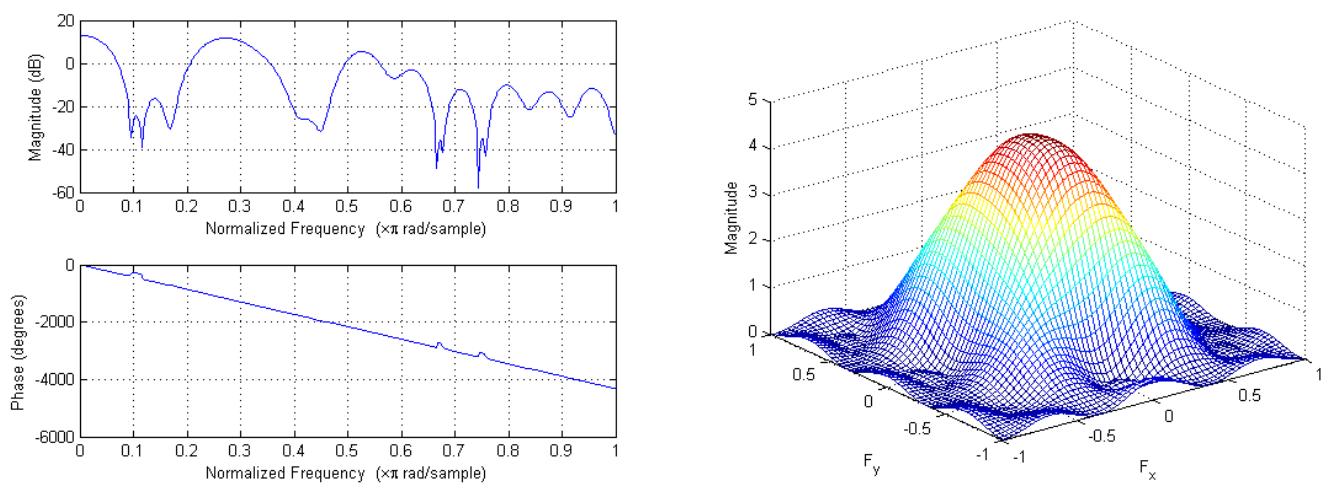

(a)
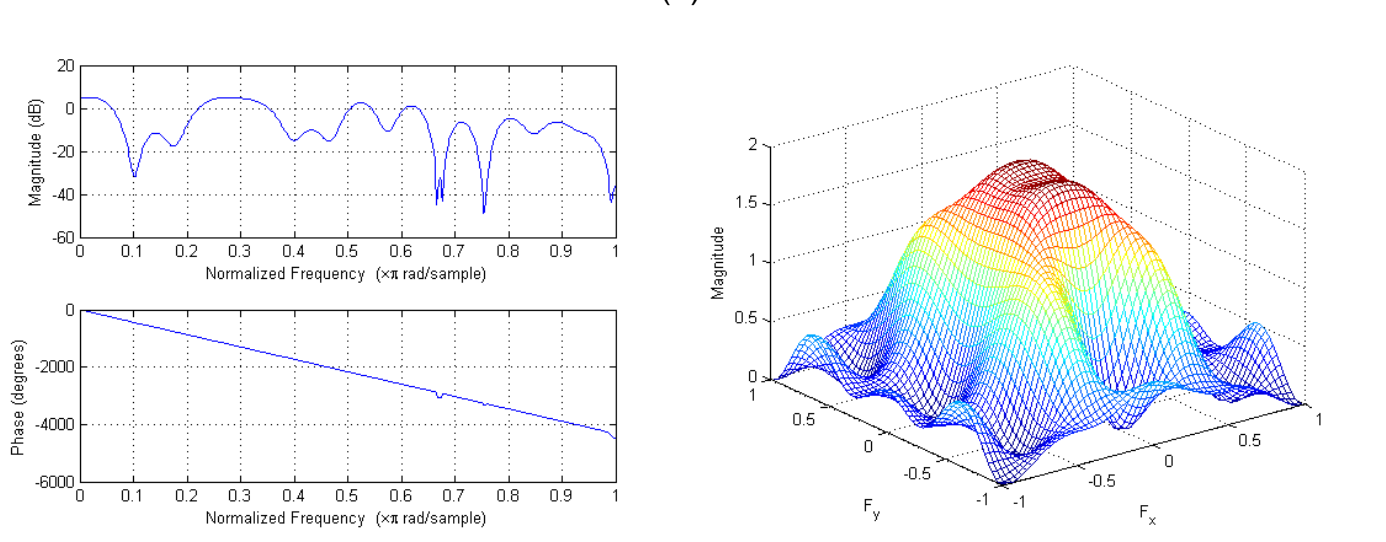

(b)
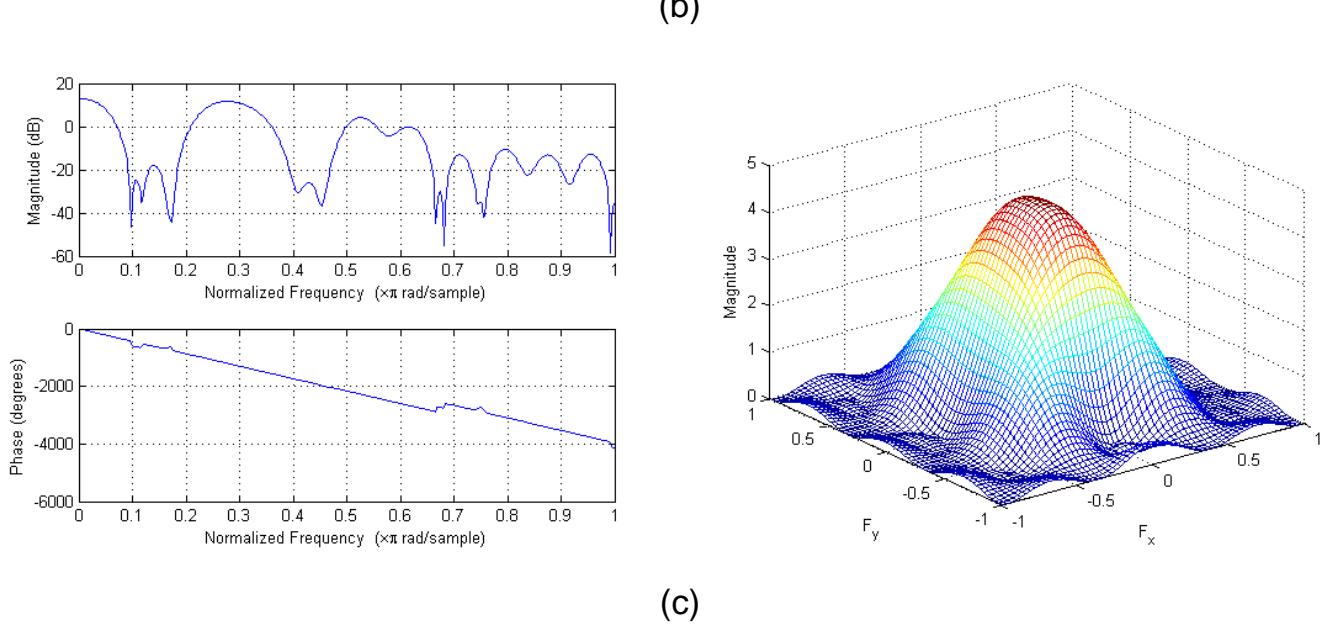

(c)

Figure 6. X-Trans CFA Pattern of $130^{\text {th }}$ LC Image, 1D Magnitude \& Phase and 2D Frequency Response: (a) Red Channel Filter, (b) Green Channel Filter, and (c) Blue Channel Filter

Figures 7 and 8 show color restored images on \#115 and \#129 images. Figures 7(a) and 8(a) show the original image and Figs. 7(b) and 8(b) show color restored image. Figures 7 (c) and 8(c) show the difference image between the original and the color restored image. 


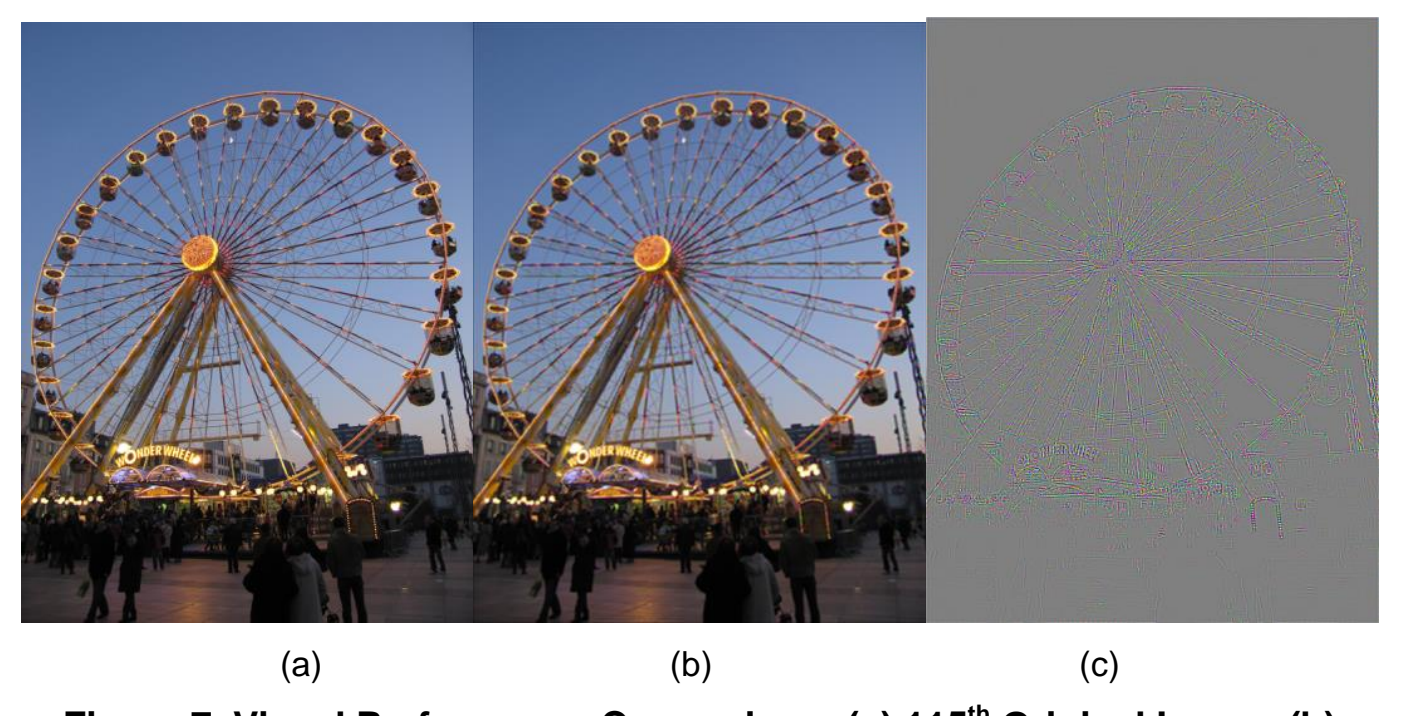

Figure 7. Visual Performance Comparison: (a) $115^{\text {th }}$ Original Image, (b) Restored Image, and (c) Difference Between the Original and the Restored
Image

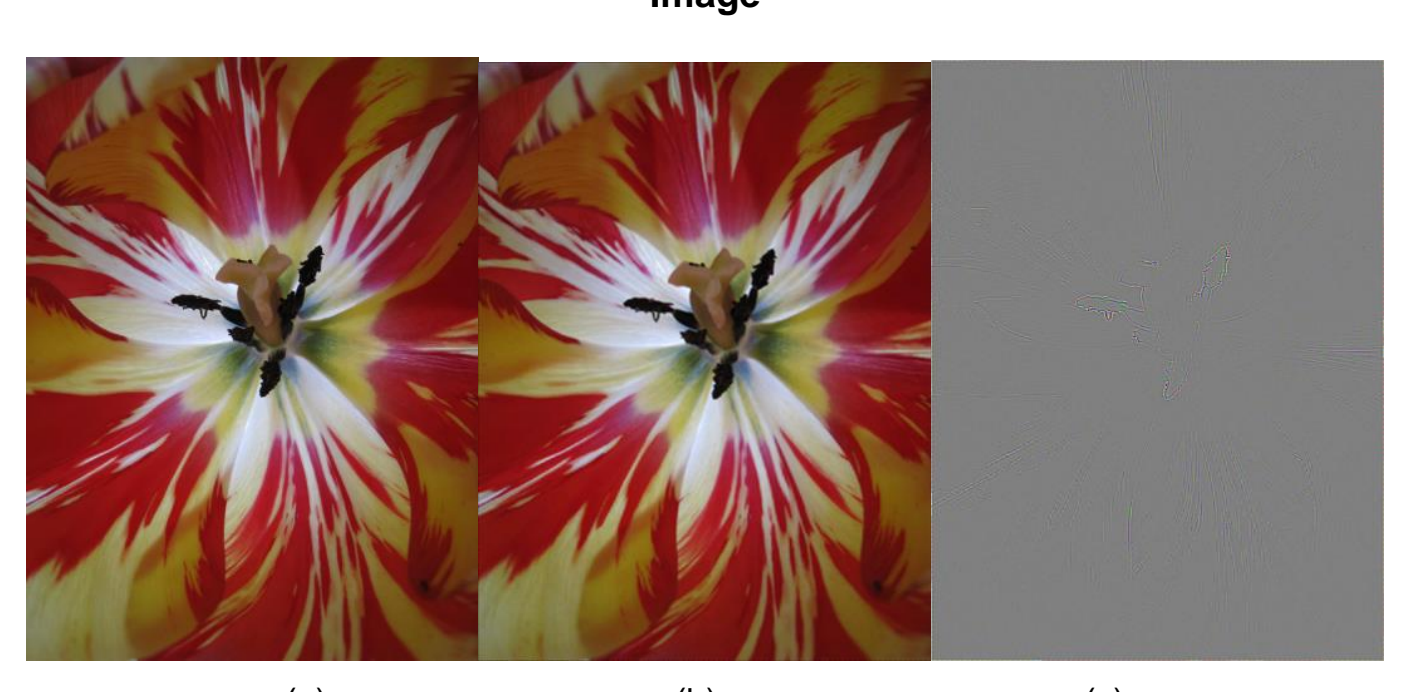

(a)

(b)

(c)

Figure 8. Visual Performance Comparison: (a) $129^{\text {th }}$ Original Image, (b) Restored Image, and (c) Difference Between the Original and the Restored Image

Figure 9 shows subjective performance comparison images. Three top images are origina red channel, green channel, and blue channel images, and three images in the middle are restored images, and three bottom images are difference images between the original and the restored images. 


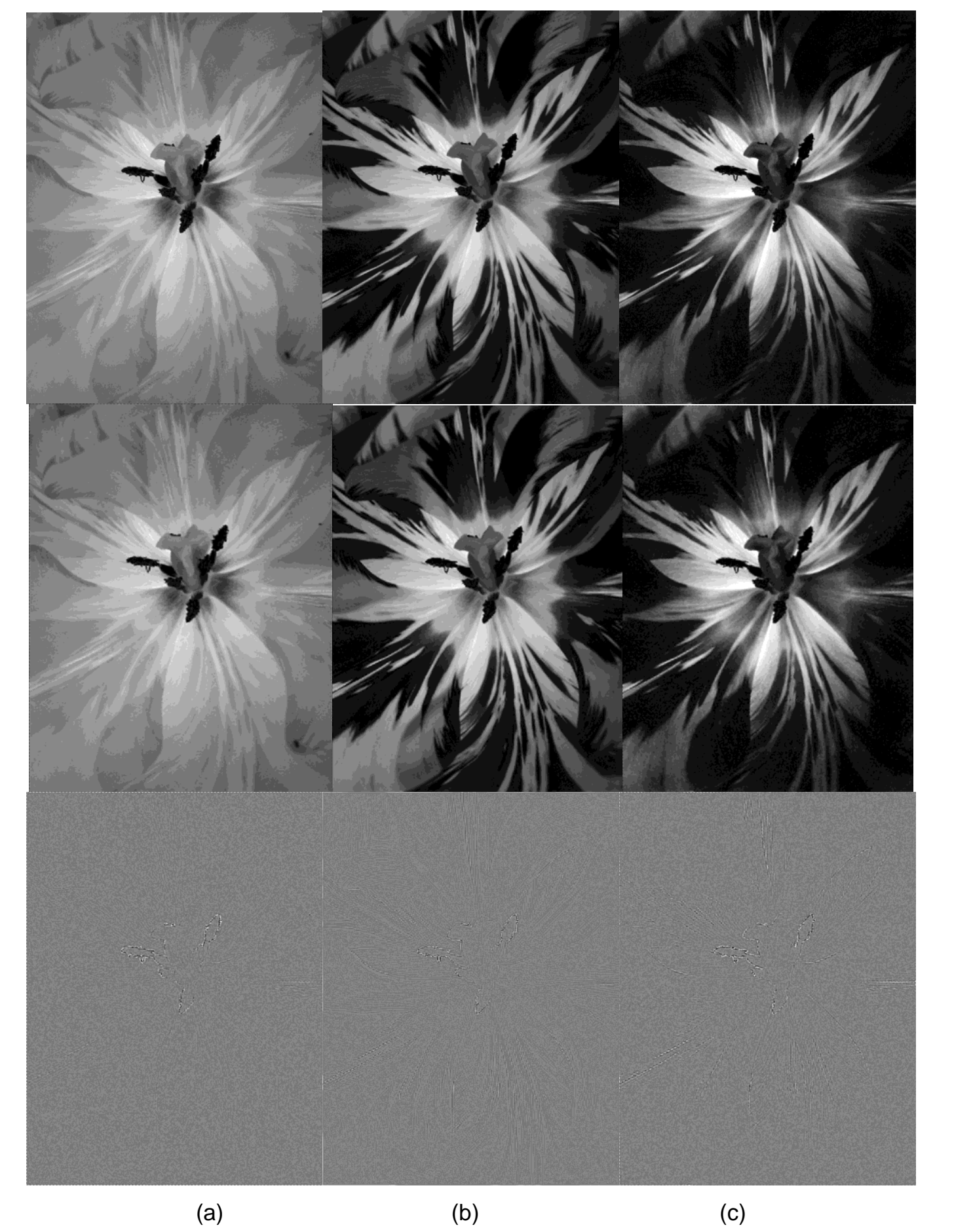

figure 9. Visual Performance Comparison, Original Image (Up), Restored Image (Middle), Difference Between Original and Restored Images (Down): (a) Red Channel, (b) Green Channel, and (c) Blue Channel 
Tables 1 and 2 show objective performance results. Tables 1 and 2 show the MSE and PSNR results in terms of red channel, green channel, blue channel, and color results.

Table 1. MSE Results For Restored X-Trans CFA Pattern Images

\begin{tabular}{|c|c|c|c|c|}
\hline & MSE $_{\text {Red }}$ & MSE $_{\text {Gren }}$ & MSE $_{\text {Blue }}$ & CMSE \\
\hline 111 & 196.826 & 133.039 & 182.198 & 170.688 \\
\hline 112 & 121.992 & 86.697 & 109.580 & 106.090 \\
\hline 113 & 249.109 & 158.041 & 195.099 & 200.749 \\
\hline 114 & 96.912 & 62.564 & 65.756 & 75.078 \\
\hline 115 & 172.028 & 145.537 & 233.618 & 183.728 \\
\hline 116 & 83.745 & 59.681 & 95.536 & 79.654 \\
\hline 117 & 163.471 & 123.475 & 132.467 & 139.804 \\
\hline 118 & 241.452 & 160.777 & 217.251 & 206.493 \\
\hline 119 & 43.233 & 28.935 & 32.988 & 35.052 \\
\hline 120 & 106.032 & 65.910 & 94.336 & 88.760 \\
\hline 121 & 243.488 & 152.449 & 235.636 & 210.524 \\
\hline 122 & 114.793 & 76.622 & 124.812 & 105409 \\
\hline 123 & 74.115 & 26.064 & 52.238 & 50.806 \\
\hline 124 & 29.551 & 31.272 & 52.960 & 37.927 \\
\hline 125 & 137.100 & 85.156 & 146.884 & 123.047 \\
\hline 126 & 12.412 & 9.991 & 13.470 & 11.958 \\
\hline 127 & 220.919 & 168.171 & $\mathbf{2 2 8 . 8 7 9}$ & 205.990 \\
\hline 128 & 69.962 & 55.836 & 36.458 & 54.085 \\
\hline 129 & 10.013 & 13.541 & 25.925 & 16.493 \\
\hline 130 & 23.968 & 19.653 & 42.066 & 28.562 \\
\hline avg. & 120.556 & 83.171 & 115.908 & 106.545 \\
\hline
\end{tabular}

Table 2. PSNR Results for Restored X-Trans CFA Pattern Images

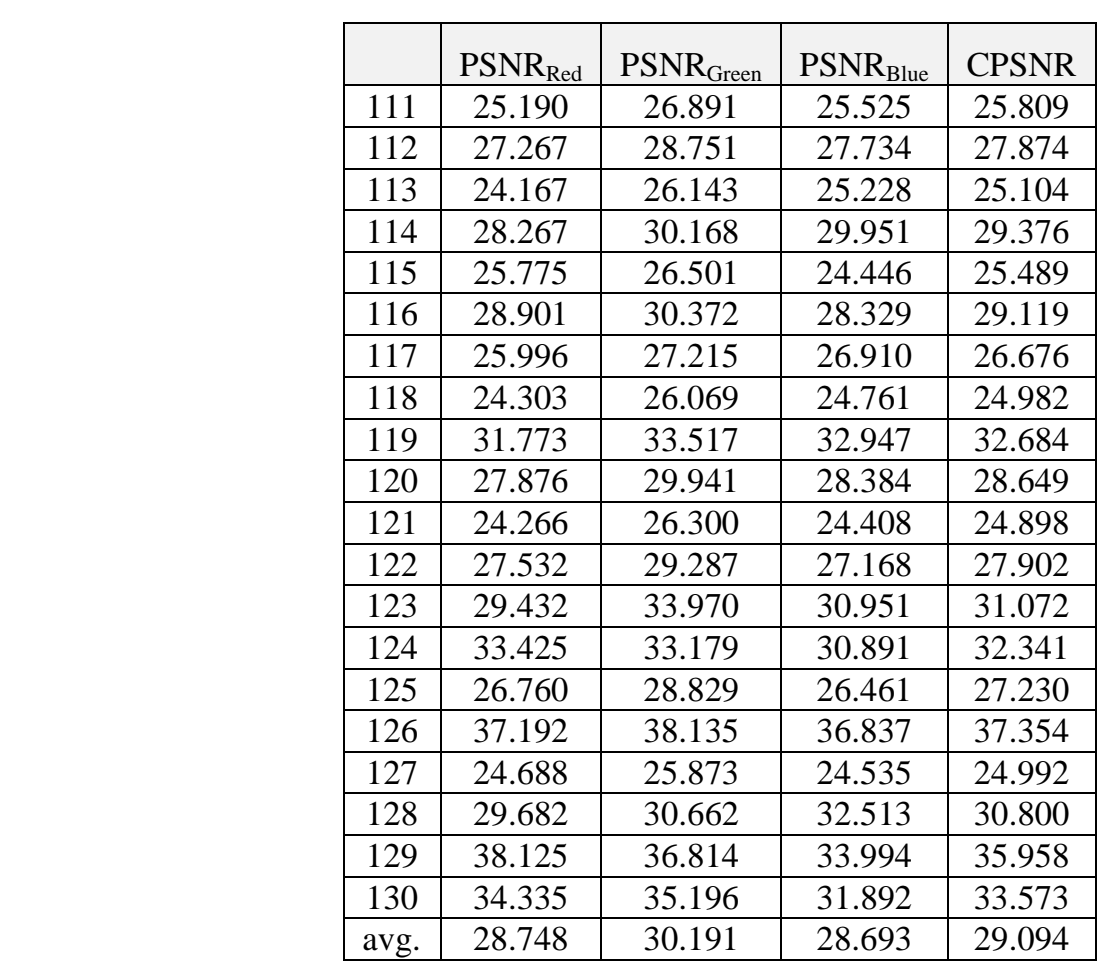




\section{Conclusions}

In this paper, we investigated X-Trans CFA pattern and its corresponding demosaicking algorithm. To solve two main issues in digital camera, color filtering approach and filter design approach were studied. To this end, a new filter based upsampling method was presented. By applying filters designed for X-Trans CFA pattern, we could achieve satisfactory objective and subjective performance.

\section{Acknowledgements}

This work was supported by the National Research Foundation of Korea(NRF) Grant funded by the Korean Government (2015R1D1A1A01058171). This paper is a revised and expanded version of a paper entitled "Color Restoration Method in a Patterned Image" presented at UCMA2016.

\section{References}

[1] K. T. Mullen, "The contrast sensitivity of human colour vision to red-green and blue-yellow chromatic grating", Journal of Physiology, vol. 359, (1995), pp. 381-400.

[2] B. S. Hur and M. G. Kang, "High definition color interpolation scheme for progressive sean CCD image sensor", IEEE Transactions on Consumer Electronics, vol. 47, no. 1, (2001), pp. 179-186.

[3] S. C. Pei and I. K. Tam, "Effective color interpolation in COD color filter array using signal correlation", IEEE Int. Conf. Image Processing, vol. 3, (2000).

[4] D. Alleysson, S. Süsstrunk, and J. Hérault, "Linear demosaieing inspired by the human visual system," IEEE Trans. Image Processing, vol. 14, no. 4, (2005), pp. 439-449.

[5] Available, http://fujifilm-x.com/x-pro1/en/about/sensor/index.html

[6] B. K. Gunturk, Y. Altunbasak and R. M. Mersereau, "Color plane interpolation using alternating projections", IEEE Transactions on Image Processing, vol. 11, no. 9, (2002).

[7] B. E. Bayer, "Color imaging array", U.S. Patent 3 971065, (1976).

[8] J. Saragih, "Principal regression analysis, IEEE CVPR2011, (2011), pp. 2881-2888.

[9] The LC image dataset, http://ww

[10] X. Zhang and B. A. Wandel1, "A spatial extension of CIELAB for digital color image reproduction", J. Soc. Inf. Display, vol. 5, no. 1, (1997), pp.61-67.

[11] R. Kimmel, "Demosaicking. Image reconstruction from color CCD samples", IEEE Trans. Image Processing, vol. 7, no 3. (999), pp. 1221-1228.

[12] G. Jeon, M. Anisêtti, D. Kim, V. Bellandi, E. Damiani, and J. Jeong, "Fuzzy rough sets hybrid scheme for motion and scene complexity adaptrve deinterlacing," Image Vision Computer, vol. 27, no. 4, (2009), pp. $425-436$.

[13] G. Jeon, M. Anisetti, J. Lee V Bellandi, E. Damiani, and J. Jeong, "Concept of linguistic variable-based fuzzy ensemble approach: application to interlaced HDTV sequences”, IEEE Trans. Fuzzy Systems, vol. 17, no. 6, (2009), pp. 1245-1258

[14] H. S. Malvar, L. W.He, and R. Cutler, "High-quality linear interpolation for demosaicing of Bayerpatterned color images', in Proceeding IEEE International Conference on Speech, Acoustics, and Signal Processing, (2004).

[15] X. Zhang and B. A.Wandell, "A spatial extension of CIELAB for digital color image reproduction", J. Soc. Inf. Display, vol. 5, no. 1, (1997), pp. 61-67.

[16] G. Jeon. M. Ánisetti, V. Bellandi, E. Damiani, and J. Jeong, "Designing of a type-2 fuzzy logic filter for improving edge-preserving restoration of interlaced-to-progressive conversion", Inf. Sci. vol. 179, no. 3, (2009), pp. 2194-2207.

Author

Gwanggil Jeon, received the BS, MS, and $\mathrm{PhD}$ (summa cum laude) degrees in Department of Electronics and Computer Engineering from Hanyang University, Seoul, Korea, in 2003, 2005, and 2008, respectively. From 2008 to 2009, he was with the Department of Electronics and Computer Engineering, Hanyang University, from 2009 to 2011, he was with the School of Information Technology and Engineering (SITE), University of Ottawa, as a postdoctoral fellow, and from 2011 to 2012, he was with the Graduate School of Science \& Technology, Niigata University, as an assistant professor. $\mathrm{He}$ is currently an associate professor with the Department of Embedded Systems 
Engineering, Incheon National University, Incheon, Korea. His research interests fall under the umbrella of image processing, particularly image compression, motion estimation, demosaicking, and image enhancement as well as computational intelligence such as fuzzy and rough sets theories. He was the recipient of the IEEE Chester Sall Award in 2007 and the 2008 ETRI Journal Paper Award.

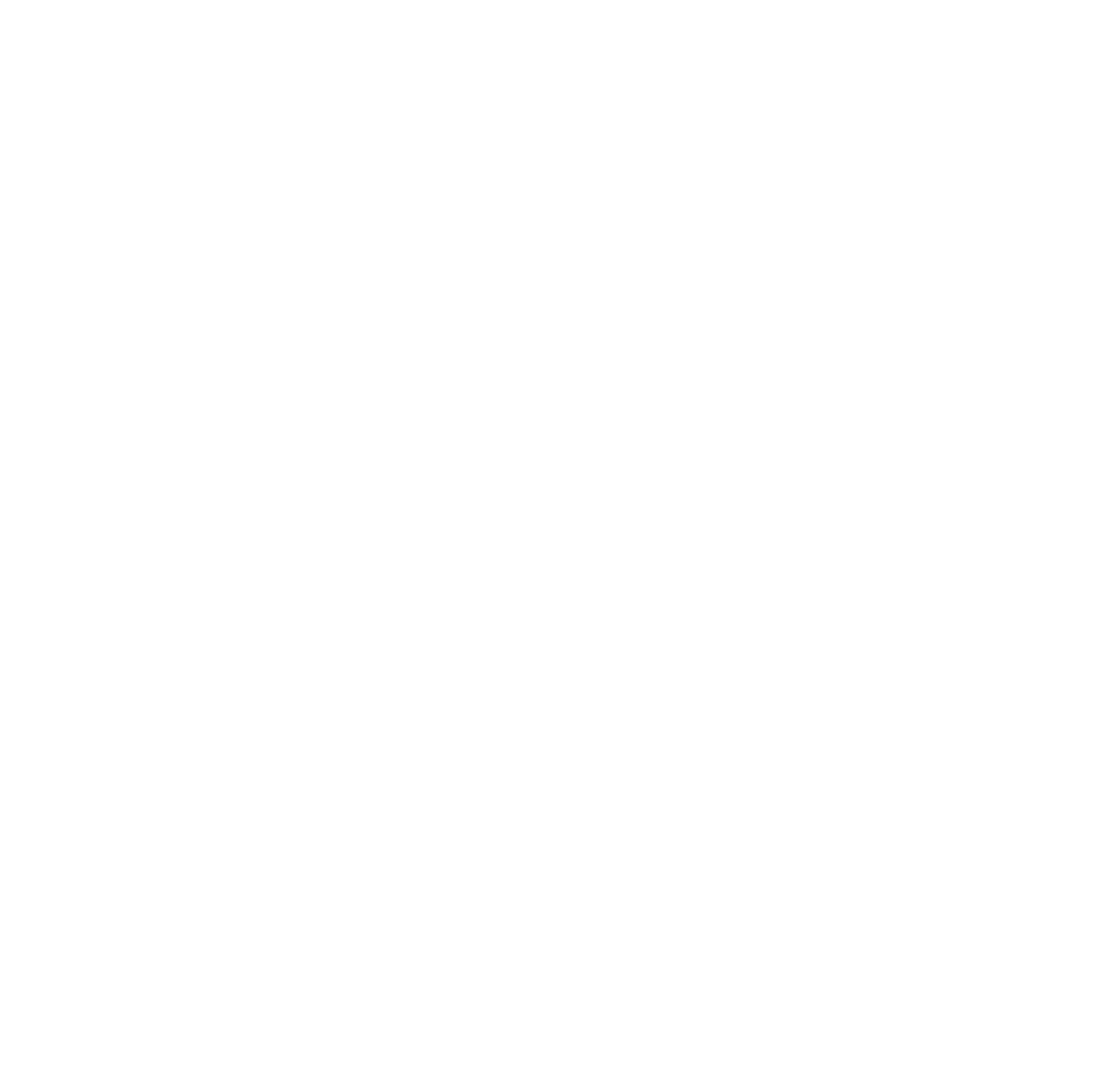

